\title{
Uso da geoestatística para caracterização da distribuição espacial de larvas de Diloboderus abderus
}

\author{
Geostatistical use for characterization of the spatial distribution of larvae of Diloboderus abderus
}

\author{
Elder Dal Prá ${ }^{I}$ Jerson Vanderlei Carús Guedes ${ }^{\mathrm{II}}$ Mariana Alejandra Cherman ${ }^{\mathrm{III}}$ \\ Affonso Hermeto Jung ${ }^{\text {II }}$ Silon Junior Procath da Silva ${ }^{\text {II }}$ Giovana Ghisleni Ribas ${ }^{\text {II }}$
}

\section{RESUMO}

No Brasil, existem registradas aproximadamente mil espécies de corós, destacando-se, dentre os de maior importância, Diloboderus abderus Sturm, 1826 (Coleoptera: Melolonthidae), pelos prejuízos que pode causar aos cultivos agrícolas e a ampla ocorrência geográfica. O trabalho teve por objetivo caracterizar, com uso da geoestatística, a distribuição espacial de larvas de D. abderus. $O$ estudo foi realizado no ano de 2009, em lavouras de aveia nos municípios de São Francisco de Assis, Cruz Alta e Lagoa Vermelha, RS. Os perímetros das áreas foram demarcados com receptor de sistema de posicionamento global, e os grides de amostragem tiveram dimensão de 70x70m. A densidade populacional foi estimada com abertura de uma trincheira em cada ponto amostral. As análises da variabilidade espacial e da dependência espacial foram feitas por meio de semivariogramas e classificadas segundo CAMBARDELLA et al. (1994). Já os mapas foram gerados a partir dos dados de contagem de larvas em campo. Os semivariogramas indicam a presença de dependência espacial nas áreas de avaliação. Os grides de amostragem mostraram-se apropriados para caracterizar a distribuição espacial de larvas de $\boldsymbol{D}$. abderus. A distribuição espacial de $\boldsymbol{D}$. abderus é agregada e seu conhecimento pode melhorar o manejo da praga.

Palavras-chave: geomática, semivariograma, krigagem, corós.

\section{ABSTRACT}

In Brazil, there are about a thousand recorded species of white grubs, and among them the most important is Diloboderus abderus Sturm, 1826 (Coleoptera: Melolonthidae), because of the damage caused to agricultural crops and the wide geographic occurrence. The study aimed to characterize, using geostatistical, the spatial distribution of larvae of $\boldsymbol{D}$. abderus. The study was conducted during 2009 in oat crops in the counties of São Francisco de Assis, Cruz Alta and Lagoa Vermelha, RS. The perimeters of the fields were delimited with global positioning system receptor, and the sampling grids dimensions were 70x70m. The population density was estimated by opening a trench in each sampling point. The analysis of spatial variability and spatial dependence were made by semivariograms and classified from CAMBARDELLA et al. (1994). Maps were generated from the counting data of larvae in the field. The semivariograms indicate the presence of spatial dependence in the assessment areas. The sampling grids proved to be suitable to characterize the spatial distribution of $\boldsymbol{D}$. abderus larvae. The spatial distribution of D. abderus is aggregated and this knowledge may improve pest management.

Key words: geomantic, kriging, semivariogram; white grubs.

\section{INTRODUÇÃO}

No Brasil, existem registradas 1.008 espécies de corós (MORÓN, 2004), sendo essa a designação popular das larvas de coleópteros edafícolas da família Melolonthidae (SALVADORI \& PEREIRA, 2006). Diloboderus abderus Sturm, 1826 (Coleoptera: Melolonthidae), vulgarmente conhecido como coródas-pastagens, é nativo de regiões com vegetação rasteira do Rio Grande do Sul, da Argentina e do Uruguai. É uma das espécies de maior importância,

IDepartamento de Defesa Fitossanitária, Universidade Federal de Santa Maria (UFSM), 97105-900, Santa Maria, RS, Brasil. Email: elderdalpra@hotmail.com. Autor para correspondência.

"UFSM, Santa Maria, RS, Brasil.

II'Universidade Federal do Paraná (UFPR), Curitiba, PR, Brasil. 
devido aos prejuízos que podem causar e pela ampla ocorrência geográfica que apresentam (GASSEN, 2001). Além disso, há muitos anos essa espécie vem sendo citada como praga de pastagens e lavouras no Rio Grande do Sul (SILVA\& LOECK, 1996). Entretanto, foi a partir da expansão de áreas cultivadas em sistema plantio direto que esse inseto-praga adquiriu maior importância econômica (GASSEN, 2001).

D. abderus é um inseto polífago que se alimenta de plantas de diversas famílias como Poaceae, Fabaceae, Asteraceae e Brassicaceae (SILVA \& COSTA, 2002). Os danos são causados, especialmente, pelas larvas de $3^{\circ}$ ínstar, que se alimentam principalmente de raízes, mas também de sementes e partes verdes das plântulas, as quais puxam para o interior do solo (SALVADORI \& PEREIRA, 2006).

Quando esses insetos-praga atingem níveis populacionais que causam dano econômico, um dos aspectos importantes para o correto emprego da aplicação de medidas de controle é a determinação da distribuição espacial, tornando-se essencial, para tanto, a escolha da técnica e da malha de amostragem, aspectos que podem interferir na eficiência do referido método (SUBRAMANYAM \& HAGSTRUM, 1996; GREGO et al., 2006).

Diversas técnicas têm sido utilizadas para determinar variações espaciais de pragas (BLACKSHAW \& ARCY-BURT, 1997; FERNANDES et al., 2002; CAMPBELL \& MULLEN, 2004; PARK \& TOLLEFSON, 2005; PARK \& TOLLEFSON, 2006). Dentre essas, a geoestatística (ELLSBURY et al., 1998; FARIAS et al., 2004; BLACKSHAW \& VERNON, 2006; FARIAS et al., 2008), que se fundamenta no princípio de que a diferença no valor de uma dada variável, em dois pontos, é dependente da distância entre esses, estando associada às técnicas usadas para analisar $\mathrm{e}$ inferir valores de uma variável distribuída no espaço e/ ou no tempo (GARCÍA, 2006). O trabalho teve por objetivo utilizar técnicas geoestatísticas para analisar a distribuição espacial de larvas de $\boldsymbol{D}$. abderus em lavouras no Estado do Rio Grande do Sul.

\section{MATERIAL E MÉTODOS}

O estudo da distribuição espacial de larvas de D. abderus foi realizado no período de 11 de julho a 27 de agosto de 2009 , em áreas de cultivo de aveia, com coordenadas $29^{\circ} 18^{\prime} 22,88^{\prime \prime} \mathrm{S}$ e $55^{\circ} 17^{\prime} 56,82^{\prime \prime} \mathrm{W}$, $28^{\circ} 31^{\prime} 29,07^{\prime \prime} \mathrm{S}$ e $53^{\circ} 41^{\prime} 46,29^{\prime \prime} \mathrm{W}, 28^{\circ} 38^{\prime} 55,01^{\prime \prime} \mathrm{S}$ e $51^{\circ} 37^{\prime} 26,03$ "W, localizadas nos municípios de São Francisco de Assis, Cruz Alta e Lagoa Vermelha, Rio Grande do Sul, respectivamente. A escolha desses municípios deu-se em função do histórico de ocorrência de larvas do coró-das-pastagens.
O perímetro das áreas foi demarcado com o auxílio de um sistema de posicionamento global (GPS) de navegação, com interface para computador-de-mão (Pocket PC). Utilizou-se o programa computacional CRCAMPEIRO 6 (GIOTTO, 2006), tecnologia móvel, para dividir as áreas experimentais em grides regulares de amostragem. Os pontos de amostragem foram localizados com o equipamento de GPS.

As áreas amostradas, dimensões do gride e número de pontos foram: 23,78ha, 70x $70 \mathrm{~m}$ e 43 pontos em São Francisco de Assis; 21,60ha, 70x70m e 43 pontos em Cruz Alta; 13,86ha, 70x70m e 30 pontos em Lagoa Vermelha. Em cada ponto amostral, foi realizada a abertura de uma trincheira, com dimensões de $0,50 \times 0,20 \times 0,20 \mathrm{~m}$ (comprimento, largura e profundidade, respectivamente), similares às propostas por SALVADORI \& PEREIRA (2006) para corós.

As larvas encontradas nas trincheiras foram conduzidas ao Laboratório de Manejo Integrado de Pragas (LabMIP) da Universidade Federal de Santa Maria e, para identificação, foram utilizados a chave pictórica de MORÓN (2006) e o guia de identificação de PEREIRA\& SALVADORI (2006).

Os dados referentes ao número de larvas foram submetidos à análise geoestatística, utilizando semivariogramas, a partir dos quais foram ajustados modelos para a interpolação das variáveis e, em etapa subsequente, foram confeccionados os mapas populacionais, gerados por krigagem ordinária. Tanto a análise geoestatística quanto os mapas populacionais foram elaborados com o programa computacional ArcGIS 9.3 (ESRI, 2004). Para a análise da dependência espacial, foi utilizada a classificação de CAMBARDELLA et al. (1994), que considera de forte dependência espacial o semivariograma que tem o valor do efeito pepita menor que $25 \%$ do patamar, moderada dependência quando o valor estiver entre 25 e $75 \%$ e de fraca dependência quando maior de $75 \%$.

Os mapas populacionais foram gerados por local de estudo, a partir dos dados da contagem de larvas de D. abderus. Esses, foram confeccionados com o número de classes variando de acordo com a densidade populacional, entretanto, com intervalo fixo (um coró por trincheira).

Em cada mapa, procedeu-se à sobreposição de planos de informação, com a inserção dos perímetros das áreas nos arquivos gerados pela krigagem. Da mesma forma, aplicou-se o algoritmo de cálculo de área, visando conhecer a área ocupada por cada classe. As coordenadas empregadas na confecção dos mapas são oriundas do datum World Geodetic System (WGS-84), no qual o sistema de coordenadas utilizado foi o Universal Transverse Mercator (UTM), estando as áreas situadas nos fusos 21 e 22S. 


\section{RESULTADOS E DISCUSSÃO}

Os semivariogramas unidirecionais ajustados pelo modelo esférico, em São Francisco de Assis, Cruz Alta e Lagoa Vermelha, são apresentados na figura $1 \mathrm{a}, \mathrm{b}$ e c, os quais o programa computacional ArcGIS (ESRI, 2004) apresenta com todos os pares da amostra, dificultando assim, a visualização do ajuste da curva teórica. No entanto, nota-se que o efeito pepita apresentou valor zero, o patamar teve valores de 1,77; 0,03 e 0,20 e o alcance foi de, aproximadamente, 195, 162 e $125 \mathrm{~m}$, respectivamente.

O alcance representa a influência dos pontos amostrados sobre sua vizinhança, variáveis localizadas a distâncias maiores que o alcance têm distribuição espacial aleatória e, por isso, são independentes entre si; portanto, o alcance pode ser usado para determinar a distância adequada entre os pontos de amostragem (VALERIANO \& PRADO, 2001; SHAO-KUI et al., 2005). Como o alcance máximo encontrado foi de $191,26 \mathrm{~m}$, a dimensão do gride amostral utilizada $(70 \times 70 \mathrm{~m})$ foi adequada e possibilitou a correta detecção da distribuição espacial de larvas de $\boldsymbol{D}$. abderus.

A dependência espacial pode ser classificada como forte, de acordo com a classificação de CAMBARDELLA et al. (1994), pois, em todos os locais estudados, o valor do efeito pepita foi inferior a
$25 \%$ do valor do patamar. Esse resultado evidencia que as larvas de melolontídeos se distribuem de forma agregada na área. Assim, segundo LIEBHOLD et al. (1993), quando existe dependência espacial entre os pontos amostrados, a distribuição espacial dos insetos é caracterizada como agregada e, nesse caso, a geoestatística é a ferramenta mais adequada para estudar as populações de insetos.

A distribuição espacial de larvas de $\boldsymbol{D}$. abderus em São Francisco de Assis pode ser visualizada na figura 2a. A densidade populacional variou de zero a seis corós por trincheira, com população superior a uma larva por trincheira em 10,21ha, sendo predominante a classe com um até dois corós por trincheira, em 6,31ha. Em Cruz Alta e Lagoa Vermelha, a área de infestação de $\boldsymbol{D}$. abderus concentrou-se em dois locais na lavoura (Figura $2 \mathrm{be}$ c), com população variando de um a dois corós por trincheira, abrangendo $1,20 \mathrm{ha} \mathrm{e} 0,73 \mathrm{ha}$, representando em torno de 5\% do total, respectivamente.

A infestação de $\boldsymbol{D}$. abderus, nas áreas de estudo, provavelmente esteja relacionada ao longo tempo de cultivo em sistema de plantio direto adotado pelos produtores nessas lavouras. Essa espécie tem sua ocorrência relacionada a solos não revolvidos e à disponibilidade de restos culturais (SALVADORI \& PEREIRA, 2006), condições necessárias para a sua

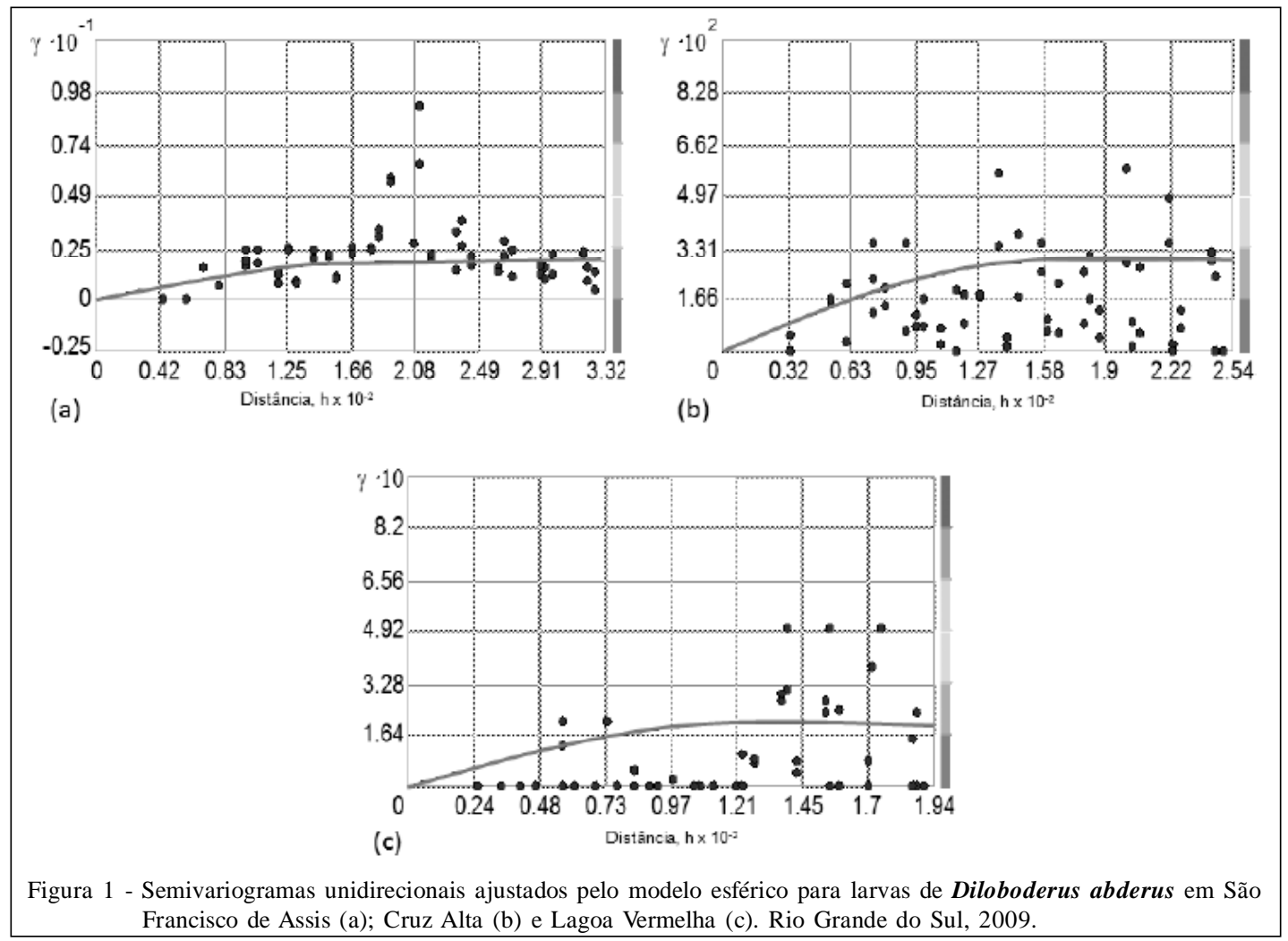

Ciência Rural, v.41, n.10, out, 2011. 


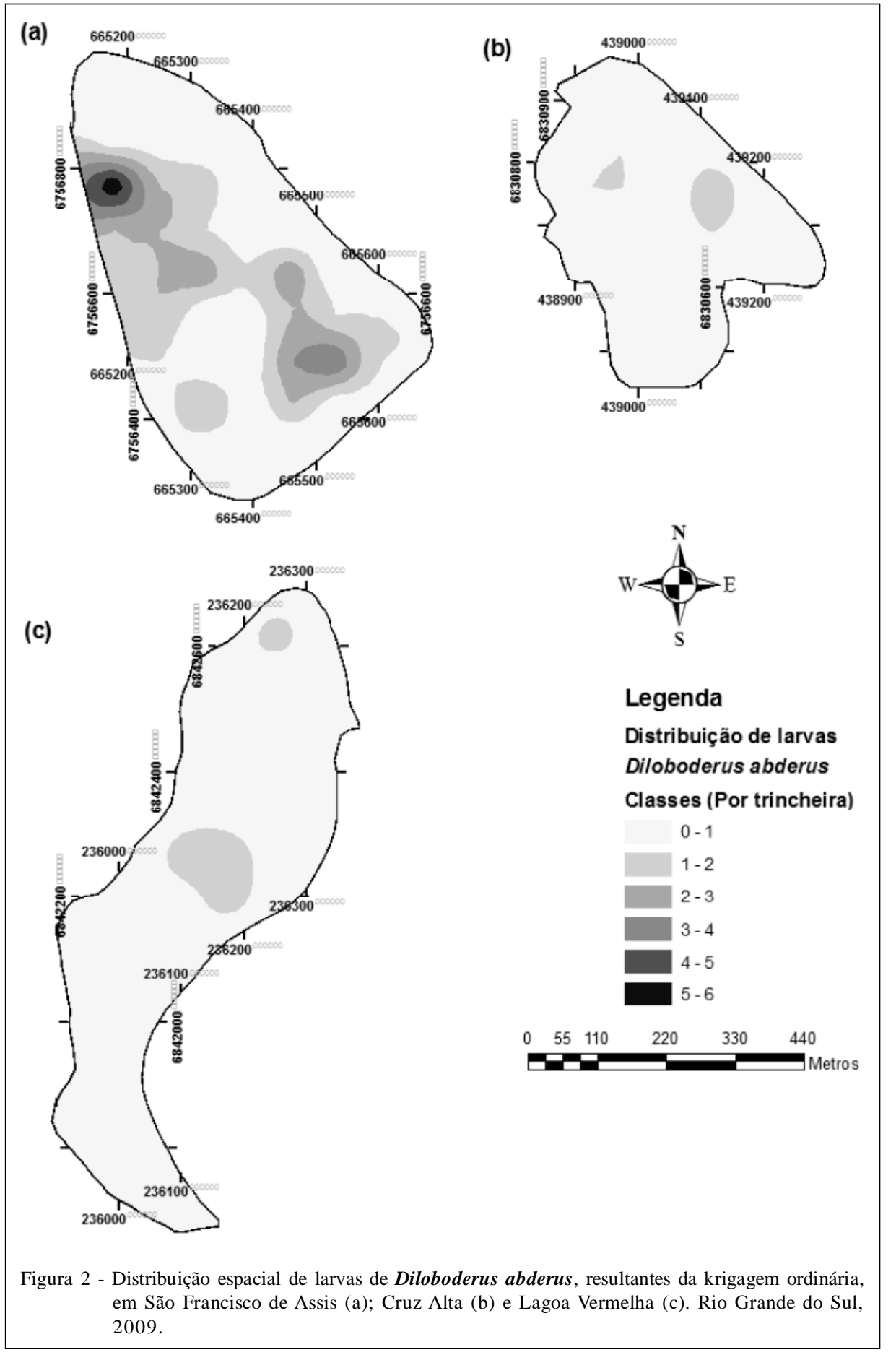

oviposição(GASSEN, 2001). Dessa forma, fica evidente a relação do plantio direto com a ocorrência desse inseto-praga.

Os mapas da distribuição espacial de $\boldsymbol{D}$. abderus nas distintas áreas demonstraram o comportamento agregado dessa espécie, nos quais é possível visualizar a distribuição em manchas ou "reboleiras" de infestação (Figura 2a, b e c). Tal observação concorda com o exposto por OLIVEIRA et al. (2005), que mencionam a distribuição espacial desse grupo de insetos como agregada, caracterizada pela maior densidade de espécimes em determinados locais 
da área. Essa distribuição determina que a amostragem deva ser eficiente o bastante, de modo a detectar as manchas de infestação.

Conhecer a dinâmica espaço-temporal dos insetos em ecossistemas agrícolas é importante para o desenvolvimento de estratégias de manejo e redução do uso de pesticidas (BLACKSHAW \& VERNON, 2006). Da mesma forma, pode proporcionar uma tomada de decisão melhor embasada, pela maior quantidade de informações obtidas e, como consequência, realizar o controle somente nas áreas com infestação. Além disso, GASSEN (2001) e SALVADORI \& PEREIRA (2006) mencionam que, para evitar o agravamento e a expansão dos danos na lavoura e para minimizar o custo, o controle pode ser realizado apenas nas reboleiras em que ocorre o ataque.

As informações apresentadas nesse estudo demonstraram a viabilidade do uso da geoestatística no estudo da distribuição espacial de larvas de $\boldsymbol{D}$. abderus, bem como na definição de espaçamentos de grides de amostragem para essa espécie. No futuro, os mapeamentos de larvas de melolontídeos deverão considerar os modelos de dependência espacial, caracterizados pelos semivariogramas apresentados nesse trabalho. Ainda, o conhecimento da distribuição espacial dos insetos, além de grides seguros para sua correta detecção, deve ser considerado como parte fundamental na elaboração e execução de programas de amostragem e de seu correto manejo.

\section{CONCLUSÃO}

A distribuição espacial de larvas de $\boldsymbol{D}$. abderus referendada pela dependência espacial forte e pelos mapas de distribuição é do tipo agregada. Grides de amostragem de 70x70m são apropriados para caracterizar a distribuição espacial de larvas de $\boldsymbol{D}$. abderus. Contudo, sugere-se a realização de novos estudos, com acréscimo do número de áreas e do período de amostragem, para que se tenha complementaridade dos resultados obtidos neste estudo.

\section{AGRADECIMENTOS}

Ao Conselho Nacional de Desenvolvimento Científico e Tecnológico (CNPq) e à Coordenação de Aperfeiçoamento de Pessoal de Nível Superior (CAPES), pelas bolsas concedidas.

\section{REFERÊNCIAS}

BLACKSHAW, R.P.; ARCY-BURT, S.D. Spatial distribution of bibionid larvae in agricultural grassland. Entomologia Experimentalis et Applicata, v.84, n.1, p.17-25, 1997.
Disponível em: <http://onlinelibrary.wiley.com/doi/10.1046/ j.1570-7458.1997.00193.x/pdf>. Acesso em: 15 nov. 2009. doi: $10.1046 / \mathrm{j} .1570-7458.1997 .00193 . x$.

BLACKSHAW, R.P.; VERNON, R.S. Spatio temporal stability of two beetle populations in non-farmed habitats in an agricultural landscape. Journal of Applied Ecology, v.43, n.4, p.680-689, 2006. Disponível em: <http:// onlinelibrary.wiley.com/doi/10.1111/j. 1365 2664.2006.01167.x/pdf>. Acesso em: 15 nov. 2009. doi: 10.1111/j.1365-2664.2006.01167.x.

CAMBARDELLA, C.A. et al. Field scale variability of soil properties in central Iowa soils. Soil Science Society of America Journal, v.58, n.6, p.1501-1511, 1994. Disponível em: <http://ddr.nal.usda.gov/bitstream/10113/18168/1/ IND20518015.pdf>. Acesso em: 21 ago. 2009.

CAMPBELL, J.F.; MULLEN, M.A. Distribution and dispersal behavior of Trogoderma variable and Plodia interpunctella outside a food processing plant. Journal of Economic Entomology, v.97, n.4, p.1455-1464, 2004. Disponível em: <http://www.bioone.org/doi/pdf/10.1603/0022-049397.4.1455>. Acesso em: 08 fev. 2010. doi: 10.1603/00220493-97.4.1455

ELLSBURY, M.M. et al. Geostatistical characterization of the spatial distribution of adult corn rootworm (Coleoptera: Chrysomelidae) emergence. Environmental Entomology, v.27, n.4, p.910-917, 1998.

ESRI. Redlands: Environmental Systems Research Institute. ArcGIS, 2004.

FARIAS, P.R.S. et al. Geostatistical characterization of the spatial distribution of Xylella fastidiosa sharpshooter vectors on citrus. Neotropical Entomology, v.33, n.1, p.13-20, 2004. Disponível em: <http://www.scielo.br/pdf/ne/v33n1/19389.pdf〉. Acesso em: 13 mar. 2009. doi: 10.1590/S1519566X2004000100004.

FARIAS, P.R.S. et al. Spatial analysis of the distribution of Spodoptera frugiperda (Lepidoptera: Noctuidae) and losses in maize crop productivity using geostatistics. Neotropical Entomology, v.37, p.321-327, 2008. Disponível em: <http:/ /www.scielo.br/pdf/ne/v37n3/a12v37n3.pdf $>$. Acesso em: 13 mar. 2009. doi: 10.1590/S1519-566X2008000300012.

FERNANDES, M.G. et al. Distribuição espacial de Spodoptera frugiperda (J. E. SMITH, 1797) (LEPIDOPTERA, NOCTUIDAE) em Algodoeiro. Revista Brasileira de Agrociência, v.8, n.3, p.203-211, 2002. Disponível em: <http://www.ufpel.tche.br/faem/agrociencia/v8n3/ artigo06.pdf>. Acesso em: 16 jun. 2009. doi: 10.1590/S1519566X2008000300012.

GARCÍA, F.J.M. Analysis of the spatio-temporal distribution of Helicoverpa armigera $\mathrm{Hb}$. in a tomato field using a stochastic approach. Biosystems Engineering, v.93, n.3, p.253-259, 2006. Disponível em: <http://people.oregonstate.edu/ kaserjo/ Garcia2006.pdf >. Acesso em: 06 jun. 2009. doi: 10.1016/ j.biosystemseng.2005.12.011.

GASSEN, D.N. Manejo de Diloboderus abderus em lavouras e pastagens. In: ROSSELO, R.D. Siembra directa en el cono sur. Montevideo: PROCISUR, 2001. p.73-182. 
GIOTTO, E. CR CAMPEIRO 6 - Sistema de gerenciamento de propriedade rural.

GREGO, C.R. et al. Spatial distribution of Pseudaletia sequax Franclemlont in triticale under no-till management. Scientia Agricola, v.63, n.4, p.321-327, 2006. Disponível em: <http:/ /www.scielo.br/pdf/sa/v63n4/a02v63n4.pdf>. Acesso em: 06 jun. 2009. doi: 10.1590/S0103-90162006000400002.

LIEBHOLD, A.M. et al. Geostatistic and geographic information system in applied insect ecology. Annual Review of Entomology, v.38, p.303-327, 1993.

MORÓN, M.A. Melolontídeos edafícolas. In: SALVADORI, J.R. et al. (Org.). Pragas de solo no Brasil. Passo Fundo: Embrapa Trigo; Dourados: Embrapa Agropecuária Oeste; Cruz Alta: Fundacep Fecotrigo, 2004. p.133-166.

MORÓN, M.A. Clave para la identificación de los principales géneros de Coleoptera Melolonthidae (Scarabaeidae-pelurosciti) de Argentina con larvas edafícolas. [S.1.:s.n.], 2006. 50p.

OLIVEIRA, C.M. et al. Análise da distribuição espacial de corós na cultura da soja em plantio direto no Cerrado. In: SIMPÓSIO INTERNACIONAL DE AGRICULTURA DE PRECISÃO, 3., 2005, Sete Lagoas. Anais... Sete Lagoas: Embrapa Milho e Sorgo, 2005. 4p.

PARK, Y.L.; TOLLEFSON J.J. Characterization of the spatial dispersion of corn root injury by corn rootworms (Coleoptera: Chrysomelidae). Journal of Economic Entomology, v.98, n.2, p.378-383, 2005. Disponível em: <http://www.bioone.org/ doi/pdf/10.1603/0022-0493-98.2.378>. Acesso em: 09 jun. 2009. doi: $10.1603 / 0022-0493-98.2 .378$

PARK, Y.L.; TOLLEFSON J.J. Spatio-temporal dynamics of corn rootworm, Diabrotica spp., adults and their spatial association with environment. Entomologia Experimentalis et Applicata, v.120, p.105-112, 2006. Disponível em: <http:/ /onlinelibrary.wiley.com/doi/ $10.1111 /$ j. 1570 - 7458.2006.00428.x/pdf>. Acesso em: 12 jul. 2009. doi: 10.1111/j.1570-7458.2006.00428.x.

PEREIRA, P.R.V.S.; SALVADORI, J.R. Guia para identificação de corós rizófagos (Coleoptera: Scarabaeoidea: Melolonthidae) comumente encontrados em cereais de inverno, milho e soja no norte do Rio Grande do Sul. Passo Fundo: Embrapa Trigo, 2006. 12p. (Comunicado Técnico, 204).

SALVADORI, J.R.; PEREIRA, P.R.V.S. Manejo integrado de corós em trigo e culturas associadas. Passo Fundo: Embrapa Trigo, 2006. 9p. (Comunicado Técnico, 203).

SHAO-KUI, G. et al. Spatial heterogeneity and population risk analysis of cotton bollworm, Helicoverpa armigera, in China. Insect Science, v.12, p.255-262, 2005. Disponível em: <http:/ /onlinelibrary.wiley.com/doi/ $10.1111 /$ j. 1005 295X.2005.00032.x/pdf>. Acesso em: 01 mar. 2009. doi: 10.1111/j.1005-295X.2005.00032.x.

SILVA, M.T.B.; COSTA, E.C. Nível de controle de Diloboderus abderus em aveia preta, linho, milho e girassol. Ciência Rural, v.32, n.1, p.7-12, 2002. Disponível em: <http:// www.scielo.br/pdf/cr/v32n1/a02v32n1.pdf>. Acesso em: 03 dez. 2008. doi: 10.1590/S0103-84782002000100002.

SILVA, M.T.B.; LOECK, A.E. Ciclo evolutivo e comportamento de Diloboderus abderus Sturm (Coleoptera: Melolonthidae) em condições de plantio direto. Anais da Sociedade Entomológica do Brasil, v.25, n.2, p.329-337, 1996.

SUBRAMANYAM, B.H.; HAGSTRUM, D.W. Integrated management of insects in stored products. New York: M. Dekker, 1996. 426p.

VALERIANO, M.M.; PRADO, H. Técnicas de geoprocessamento e de amostragem para o mapeamento de atributos anisotrópicos do solo. Revista Brasileira de Ciência do Solo, v.25, p.997-1005, 2001. 